\title{
Validez del hallazgo de nódulos en la colonoscopia para el diagnóstico de hiperplasia nodular linfoide en niños
}

\section{Validity of nodules detected at colonoscopy for the diagnosis of nodular lymphoid hyperplasia in children}

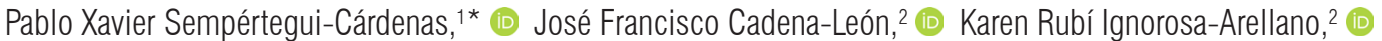 \\ Erick Manuel Toro-Monjaraz, ${ }^{2}$ (c) Flora Elva Zárate-Mondragón, ${ }^{3}$ (1) Roberto Cervantes-Bustamante, ${ }^{2}$ (C) Jaime Alfonso Ramírez-Mayans, ${ }^{2}$ (1) \\ Celso Tomás Corcuera-Delgado, ${ }^{4}$ Laura Becerril-Cholula. ${ }^{4}$ (D)
}

\author{
GacCeso abierto \\ Citación: \\ Sempértegui-Cárdenas PX, Cadena-León JF, \\ Ignorosa-Arellano KR, Toro-Monjaraz EM, \\ Zárate-Mondragón FE, Cervantes-Bustamante \\ R, Ramírez-Mayans JA, Corcuera-Delgado \\ CT, Becerril-Cholula L. Validez del hallazgo de \\ nódulos en la colonoscopia para el diagnóstico de \\ hiperplasia nodular linfoide en niños. Rev Colomb \\ Gastroenterol. 2021;36(1):24-29. https://doi. \\ org/10.22516/25007440.564 \\ Mg. Médico Pediatra, especialista en \\ Gastroenterología y Nutrición Pediátrica. \\ Departamento de Gastroenterología y Nutrición, \\ Instituto Nacional de Pediatría, Ciudad de \\ México, México. \\ Médico Pediatra, especialista en \\ Gastroenterología y Nutrición Pediátrica. \\ Departamento de Gastroenterología y Nutrición, \\ Instituto Nacional de Pediatría, Ciudad de \\ México, México. \\ 3 PhD. Médico Pediatra, especialista en \\ Gastroenterología y Nutrición Pediátrica. \\ Departamento de Gastroenterología y Nutrición, \\ Instituto Nacional de Pediatría, Ciudad de \\ México, México. \\ Médico especialista en anatomía patológica. \\ Servicio de Anatomía Patológica, Instituto \\ Nacional de Pediatría, Ciudad de México, México. \\ El presente trabajo fue presentado únicamente \\ como cartel en la Semana Nacional de \\ Gastroenterología de México. Cancún, 16-20 de \\ noviembre de 2019 \\ ${ }^{*}$ Correspondencia: \\ Pablo Xavier Sempértegui-Cárdenas. \\ troverospa@gmail.com \\ Fecha recibido: $11 / 05 / 20$ \\ Fecha aceptado: 21/07/20
}

\section{Resumen}

Introducción: la hiperplasia nodular linfoide del colon se define como $>10$ nódulos linfoides visibles en colonoscopia. No existen estudios de su validez al compararlo con la histopatología. Objetivos: determinar la validez del hallazgo de nódulos en colonoscopia para el diagnóstico de hiperplasia nodular linfoide. Material y métodos: estudio prospectivo de prueba diagnóstica. Se incluyeron colonoscopias realizadas consecutivamente de 2014 al 2018 con equipos Olympus PCFQ150Al y GIFXP150N con obtención de biopsias. El criterio endoscópico fue la presencia de > 10 nódulos de 2 a $10 \mathrm{~mm}$ y el criterio histológico fue hiperplasia de folículos linfoides y mantos de linfocitos en lámina propia o submucosa. Los datos se analizaron en Epidat3.1. Se obtuvo la sensibilidad (S), especificidad (E), valor predictivo positivo (VPP) y negativo (VPN), y coeficientes de probabilidad positivo (LR+) y negativo (LR-) con sus intervalos de confianza. Resultados: se incluyeron 327 colonoscopias, la mediana de edad fue de 84 meses. La principal indicación para la colonoscopia fue sangrado digestivo bajo (38,8 \%). El hallazgo de nódulos se encontró en el $21 \%$ y el sitio de mayor frecuencia fue el colon total (46\%), mientras que por histopatología se encontró hiperplasia nodular linfoide en el $38 \%$. El hallazgo de nódulos obtuvo una S de $32 \%$ (intervalo de confianza [IC] del $95 \%$ : 24-140), E de $84 \%$ (IC $95 \%$ : 79-89), VPP de $56 \%$ (IC $95 \%$ : 44-68), VPN de $67 \%$ (IC $95 \%: 61-72$ ), LR+ de 2,04 (IC 95\%: 1,4-3) y LR- de 0,8 (IC $95 \%$ : 0,8-0,9). Conclusiones: la validez diagnóstica del hallazgo de nódulos en colonoscopia para hiperplasia nodular linfoide es pobre, por lo que la toma de biopsia debe recomendarse siempre.

\section{Palabras clave}

Niños, colonoscopia, hiperplasia nodular linfoide, sensibilidad, especificidad.

\begin{abstract}
Introduction: Nodular lymphoid hyperplasia of the colon is characterized by the presence of $>10$ lymphoid nodules visible in colonoscopy. There are no studies that confirm their validity when compared with histopathology. Objective: To determine the validity of nodules detected at colonoscopy for the diagnosis of nodular lymphoid hyperplasia in children. Materials and methods: Prospective study of diagnostic test accuracy. Colonoscopies performed consecutively from 2014 to 2018 using Olympus PCFQ150AI and GIFXP150N biopsy machines were included. The endoscopic criterion was the presence of $>10$ nodules from 2 to $10 \mathrm{~mm}$ of diameter, while the histological criterion was presence of follicular lymphoid hyperplasia and lymphocyte mantles in lamina propia or submucosa. Data were analyzed in Epidat3.1. Sensitivity (SE), specificity (SP), positive predictive value (PPV), negative predictive value (NPV), positive likelihood ratio (LR+) and negative likelihood ratio (LR-) were obtained with their corresponding confidence intervals. Results: 327 colonoscopies were included; the median age was 84 months. The main indication for colonoscopy was lower gastrointestinal bleeding $(38.8 \%)$. Nodules were found in $21 \%$ of the patients, predominantly throughout the whole colon (46\%), whereas histopathology found nodular lymphoid hyperplasia in $38 \%$. SE for the finding of nodules was $32 \%$ (95\% confidence interval [Cl]: 24-140), SP was $84 \%$ (95\% Cl: $79-89)$, PPV was $56 \%$ (95\% Cl: 44-68), NPV was $67 \%$ (95\% Cl: 61-72), LR+ was 2.04 (95\% Cl: 1.4-3) and LR- was 0.8 (95\% Cl: 0.8-0.9). Conclusions: The validity of the presence of nodules on colonoscopy for the diagnosis of nodular lymphoid hyperplasia is poor, so biopsy should always be performed.
\end{abstract}

Keywords

Children, Colonoscopy, Nodular lymphoid hyperplasia, Sensitivity, Specificity. 


\section{INTRODUCCIÓN}

La hiperplasia nodular linfoide del colon se define como un grupo de $>10$ nódulos linfoides observados durante la colonoscopia (1), estos se han reportado con mayor frecuencia en niños pequeños no asociados con síntomas clínicos, en los cuales la hiperplasia nodular linfoide se considera fisiológica (1-3); sin embargo, se ha reportado en pacientes sometidos a colonoscopia por sangrado digestivo, dolor abdominal crónico (4), constipación refractaria, retardo del crecimiento o anemia, en frecuencias que varían de $12,8 \%$ (5) hasta el $49 \%$ (1). En un estudio cubano se encontró que el $63 \%$ se presentó en menores de 6 años; $33 \%$, entre 6 y 10 años; y $4 \%$, en mayores de 10 años (6).

La importancia clínica y la etiología de la hiperplasia nodular linfoide no está clara. Durante muchos años se pensó que era un hallazgo casual en la colonoscopia; sin embargo, se ha asociado con patologías infecciosas como Helicobacter pylori (7), oxiuros, ameba, Escherichia coli y Giardia lamblia (8-11); fiebre familiar del mediterráneo; inmunodeficiencias como la inmunodeficiencia común variable (12-14), deficiencia de IgA $(14,15)$, hipogammaglobulinemia $(12,13,16)$ y virus de la inmunodeficiencia humana (VIH) (17); alergia alimentaria; enfermedad inflamatoria intestinal $(1,4,5,18)$; síndrome de intestino irritable; enfermedad celíaca; enfermedad de Behçet; entre otras (5).

El diagnóstico se establece mediante endoscopia y se confirma histológicamente (19). Las características endoscópicas incluyen nódulos que varían entre 2 a $10 \mathrm{~mm}$ y pueden presentarse en el estómago, el intestino delgado (más comúnmente en el íleon terminal) y el colon/recto (20). En el colon pueden aparecer como máculas rojas, lesiones diana circunferenciales o pápulas elevadas $(21,22)$. Cuando el intestino grueso está afectado, el recto está implicado con mayor frecuencia $(23,24)$. El aspecto endoscópico puede ser sorprendentemente similar a los síndromes de poliposis (23), pero los hallazgos histológicos pueden ayudar al diagnóstico diferencial. Histológicamente, se define por la presencia de hiperplasia de folículos linfoides, centros germinales con actividad mitótica y mantos de linfocitos bien definidos localizados en lámina propia o submucosa superficial (25); puede parecerse a un linfoma clínico e histológicamente maligno (26), pero se distingue por su naturaleza polimórfica del infiltrado, la ausencia de atipia citológica significativa y la presencia de folículos reactivos dentro de la lesión (27).

La hiperplasia nodular linfoide se considera un factor de riesgo para el desarrollo de linfoma intestinal $(28,29)$ y extraintestinal $(30,31)$, adenomas y carcinomas (32), por lo que algunos autores recomiendan series baritadas y cápsulas endoscópicas seriadas (24), aunque la duración y los intervalos de dicha vigilancia no están definidos (15). No existen estudios previos que evalúen la validez diagnóstica del hallazgo de nódulos durante la colonoscopia al compararlo con la histología en el diagnóstico de hiperplasia nodular linfoide.

\section{OBJETIVO}

Determinar la validez del hallazgo de nódulos durante la colonoscopia para el diagnóstico de hiperplasia nodular linfoide en niños.

\section{MATERIAL Y MÉTODOS}

Se realizó un estudio de prueba diagnóstica de tipo prospectivo en un hospital pediátrico de referencia. Se incluyeron 327 colonoscopias realizadas de forma consecutiva durante el período del 1 de enero de 2014 al 31 de diciembre de 2018. La muestra fue calculada en Epidat 3.2 con base en la sensibilidad esperada de $40 \%-60 \%$, especificidad esperada de $70 \%-90 \%$, prevalencia de enfermedad de $30,9 \%$ (promedio de prevalencias reportadas en la literatura) $(1,5)$, intervalo de confianza (IC) de $95 \%$ y potencia de $80 \%$. Todas las colonoscopias fueron realizadas por un gastroenterólogo pediátrico entrenado en colonoscopia; en niños de más de $10 \mathrm{~kg}$ se utilizó un colonoscopio Olympus PCF-Q150AI, mientras que para niños de menor peso se utilizó un endoscopio neonatal Olympus GIF-XP150N; en todos los casos se tomaron biopsias durante la colonoscopia mediante pinza de biopsia fría con al menos 2 muestras de ciego, 2 de colon ascendente, 2 de colon transverso, 2 de descendente y 2 de rectosigmoides, las cuales se analizaron de forma ciega por 2 patólogos del departamento de histopatología del instituto en un plazo máximo de 1 semana. Los patólogos fueron validados previamente mediante concordancia interobservador con un coeficiente de Kappa de Cohen de 1,0 $(p=0,000)$. No se presentó ningún evento adverso durante la colonoscopia ni durante la toma de biopsia.

El criterio colonoscópico se definió conforme a lo publicado en la literatura con base en el hallazgo de un grupo de $>10$ nódulos de 2 a $10 \mathrm{~mm}$ visibles en cualquier sitio del intestino grueso (20). La histología se analizó mediante tinciones de hematoxilina-eosina con un microscopio óptico a 5X, 10X y 40X; el criterio histológico se definió por lo citado en la literatura con base en la presencia de hiperplasia de folículos linfoides y mantos de linfocitos bien definidos localizados en lámina propia o submucosa superficial (25).

Los datos se registraron en una base en el programa SPSS versión 22 , en el que se codificaron para su tabulación. De la variable edad se realizó una prueba de normalidad con el estadístico de Kolmogorov-Smirnov y, debido a que presentó una distribución anormal, se resumió mediante 
la mediana y los rangos intercuartílicos. De las variables cualitativas tales como intervalo de edad, sexo, indicación para la colonoscopia, hallazgos colonoscópicos, sitio de hallazgo colonoscópico y hallazgos histológicos se obtuvieron frecuencias y porcentajes. Los hallazgos colonoscópicos se dicotomizaron con base en la presencia o ausencia de nódulos visibles y los hallazgos histológicos, con base en la presencia o ausencia de hiperplasia nodular linfoide y se cruzaron en tablas de $2 \times 2$. Se comparó la proporción de hiperplasia nodular de acuerdo con la edad y la indicación para la colonoscopia mediante chi cuadrado $\left(\chi^{2}\right)$. Se utilizó el programa Epidat 3.1 para obtener sensibilidad, especificidad, valor predictivo positivo (VPP) y negativo (VPN), y coeficientes de probabilidad positivo y negativo con sus respectivos intervalos de confianza, así como el valor global de la prueba diagnóstica.

\section{RESULTADOS}

De las 327 colonoscopias realizadas, 188 fueron en hombres $(57,5 \%)$ y el resto en mujeres. La mediana de edad en el grupo fue de 84 meses ( 7 años) con un rango intercuartílico de 111 meses, un valor mínimo de 1 mes y un máximo de 216 meses.

La principal indicación para la realización de la colonoscopia fue el sangrado de tubo digestivo bajo (anemizante o no) con 127 casos (38,8\%), seguido en frecuencia por sospecha o vigilancia de enfermedad inflamatoria intestinal (17\%), sospecha o seguimiento de alergia alimentaria $(14,4 \%)$, diarrea crónica $(11,3 \%)$, vigilancia de síndrome de poliposis $(6,1 \%)$ y sospecha de pólipo rectal $(3,7 \%)$; el porcentaje restante se distribuyó en causas diversas tales como sospecha de enfermedad de injerto contra huésped, dolor abdominal crónico, enteropatía perdedora de proteínas, malformación vascular, cuerpos extraños, sospecha de cáncer de colon, entre otras.

La prevalencia del hallazgo colonoscópico de nódulos fue del 21,7\% ( $\mathrm{n}=71)$. La ubicación donde se reportaron nódulos con mayor frecuencia fue el colon total con 33 casos (46\%), seguida por afección limitada al recto (17\%), limitada al colon izquierdo (14\%) y limitada al rectosigmoides (13\%). La proporción de nódulos encontrada en hombres fue similar al de mujeres $(21,3 \%$ frente a $22,3 \%)$. Los nódulos se presentaron con mayor frecuencia a menor edad $(p=0,016)$ (Tabla 1 y Figura 1$)$.

La prevalencia de hiperplasia nodular linfoide en el reporte histopatológico fue de $38 \%(\mathrm{n}=124)$. Los porcentajes más bajos de hiperplasia nodular linfoide se registraron en pacientes sometidos a colonoscopia por invaginación intestinal recurrente, pérdida de peso inexplicable y sospecha de pseudoobstrucción (0\%), seguido en orden ascendente por vigilancia de síndromes de poliposis $(5 \%)$ y sospecha de enfermedad de injerto contra huésped (11\%). Las indicaciones por las que se encontró hiperplasia nodular linfoide con mayor frecuencia fueron sospecha de cáncer de colon, sospecha de tuberculosis intestinal y constipación refractaria $(100 \%)$, seguidas en orden descendente por sospecha de fístula rectal y sospecha de enteropatía ( $50 \%$ ), sospecha o vigilancia de enfermedad inflamatoria intestinal (49\%), sospecha de pólipo rectal (42\%) y sospecha o seguimiento de alergia alimentaria (40\%).

Tabla 1. Distribución de pacientes sometidos a colonoscopia de acuerdo con el hallazgo de nódulos, sexo y edad (2014-2018)

\begin{tabular}{|c|c|c|c|c|c|c|c|}
\hline \multirow[t]{3}{*}{ Variable } & \multicolumn{4}{|c|}{ Nódulos } & \multirow{3}{*}{$\begin{array}{c}\text { Total } \\
\mathbf{n}\end{array}$} & \multirow{3}{*}{$\underset{\text { Pearson }}{X^{2}}$} & \multirow{3}{*}{$\begin{array}{l}\text { Valor } \\
\text { de } p\end{array}$} \\
\hline & \multicolumn{2}{|c|}{ Sí } & \multicolumn{2}{|c|}{ No } & & & \\
\hline & $\mathbf{n}$ & $\%$ & $\mathrm{n}$ & $\%$ & & & \\
\hline \multicolumn{8}{|l|}{ Sexo } \\
\hline Hombre & 40 & 21,3 & 148 & 78,7 & 188 & 0,049 & 0,892 \\
\hline Mujer & 31 & 22,3 & 108 & 77,7 & 139 & & \\
\hline \multicolumn{8}{|l|}{ Edad (años) } \\
\hline$<2$ & 16 & 32,0 & 34 & 68,0 & 50 & 8,295 & $0,016^{*}$ \\
\hline $2-10$ & 40 & 24,2 & 125 & 75,8 & 165 & & \\
\hline$>10$ & 15 & 13,4 & 97 & 86,6 & 112 & & \\
\hline Total & 71 & 21,7 & 256 & 78,3 & 327 & & \\
\hline
\end{tabular}

${ }^{*}$ Existe diferencia estadísticamente significativa. Fuente: formularios de recolección. Elaboración de los autores.

De acuerdo con la indicación para la realización de colonoscopia, la frecuencia de hiperplasia nodular linfoide mostró diferencias estadísticamente significativas $(p=$ 0,038). En cuanto al diagnóstico definitivo, la patología en la que se documentó con más frecuencia la hiperplasia nodular linfoide fue la alergia alimentaria $(47 \%, \mathrm{n}=81)$, seguida en frecuencia por colitis crónica inespecífica ( $52 \%$, $\mathrm{n}=105)$, enfermedad inflamatoria intestinal $(22 \%, \mathrm{n}=27)$ y poliposis $(22 \%, \mathrm{n}=59)$; los casos de sospecha de cáncer de colon y tuberculosis intestinal fueron descartados.

Solo el $32 \%$ de los pacientes con hiperplasia nodular linfoide presentó un patrón nodular en la colonoscopia, mientras que el $84 \%$ de pacientes sin hiperplasia nodular linfoide no mostró un patrón nodular; el $56 \%$ de pacientes con un patrón nodular tuvo hiperplasia nodular linfoide y el $67 \%$ de pacientes sin patrón nodular no tuvo hiperplasia nodular linfoide; los pacientes con hiperplasia nodular tienen 2 veces más probabilidades de tener un patrón nodular en la colonoscopia, mientras que en los pacientes sin hiperplasia nodular existen 1,25 veces más probabilidades de no tener un patrón nodular (Tabla 2). La validez global del patrón nodular fue del $64 \%$. 
Tabla 2. Distribución de pacientes sometidos a colonoscopia de acuerdo con el hallazgo de nodularidad y hallazgo histopatológico de hiperplasia nodular linfoide (2014-2018)

\begin{tabular}{|c|c|c|c|c|c|c|c|c|c|}
\hline \multirow{2}{*}{$\begin{array}{l}\text { Patrón nodular } \\
\text { por colonoscopia }\end{array}$} & \multicolumn{3}{|c|}{ Hiperplasia nodular linfoide por histología } & \multirow{2}{*}{$\begin{array}{c}\mathrm{S} \\
\text { (IC } 95 \%)\end{array}$} & \multirow{2}{*}{$\begin{array}{c}\mathrm{E} \\
\text { (IC } 95 \% \text { \%) }\end{array}$} & \multirow{2}{*}{$\begin{array}{c}\text { VPP } \\
\text { (IC } 95 \%)\end{array}$} & \multirow{2}{*}{$\begin{array}{c}\text { VPN } \\
\text { (IC } 95 \%)\end{array}$} & \multirow{2}{*}{$\begin{array}{c}\text { LR+ } \\
\text { (IC } 95 \%)\end{array}$} & \multirow{2}{*}{$\begin{array}{l}\text { LR- } \\
(\text { IC } 95 \%)\end{array}$} \\
\hline & Sí & No & Total & & & & & & \\
\hline Sí & 39 & 32 & 71 & \multirow{3}{*}{$\begin{array}{c}32,31 \\
(23,8-40,7)\end{array}$} & \multirow{3}{*}{$\begin{array}{c}84,13 \\
(78,9-89,3)\end{array}$} & \multirow{3}{*}{$\begin{array}{c}56 \\
(44,1-67,9)\end{array}$} & \multirow{3}{*}{$\begin{array}{c}66,5 \\
(60,5-72,4)\end{array}$} & \multirow{3}{*}{$\begin{array}{c}2,04 \\
(1,37-3,04)\end{array}$} & \multirow{3}{*}{$\begin{array}{c}0,8 \\
(0,7-0,92)\end{array}$} \\
\hline No & 85 & 171 & 256 & & & & & & \\
\hline Total & 124 & 203 & 327 & & & & & & \\
\hline
\end{tabular}

E: especificidad; IC: intervalos de confianza; LR: razón de verosimilitud; S: sensibilidad; VPN: valor predictivo negativo; VPP: valor predictivo positivo. Fuente: formularios de recolección. Elaboración de los autores.

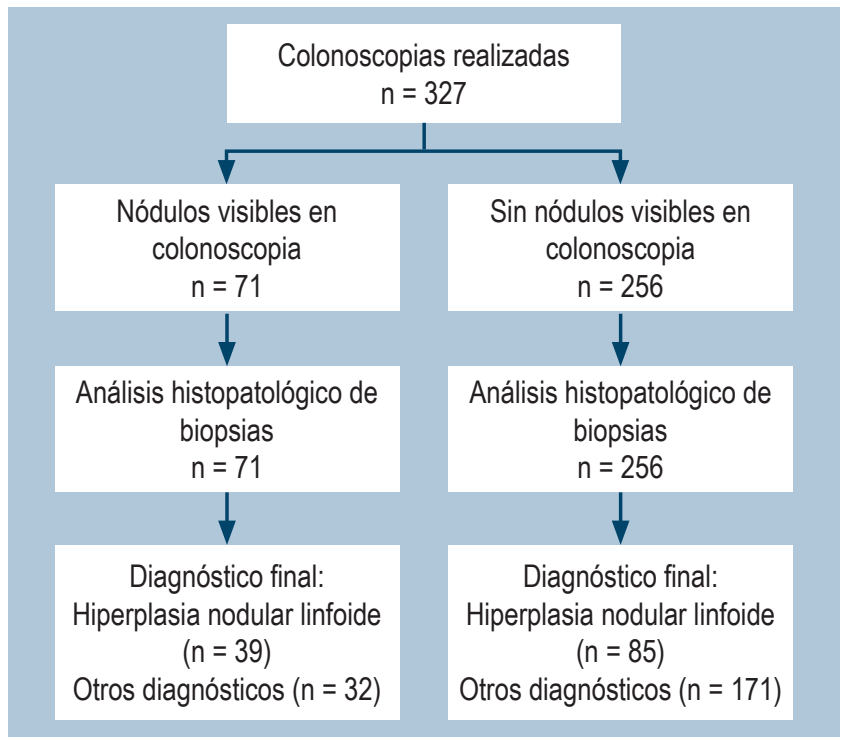

Figura 1. Flujo de participantes sometidos a colonoscopia y toma de biopsias.

\section{DISCUSIÓN}

En nuestro estudio, la principal indicación para la colonoscopia fue el sangrado de tubo digestivo bajo con un $38,8 \%$, porcentaje similar al $32 \%$ de sangrado publicado por Colon y colaboradores (4).

La prevalencia del hallazgo colonoscópico de nódulos en nuestro estudio fue similar a lo publicado por Zahmatkeshan y colaboradores (33); sin embargo, la prevalencia confirmada por histopatología en nuestro estudio fue casi del doble que el encontrado en la endoscopia, por lo que probablemente haya estado subdiagnosticada.

En la mayoría de pacientes con hallazgo de nódulos se encuentra que la afección colónica fue total, lo que no concuerda con la literatura publicada, en la que se señala que el número de nódulos en el colon es mayor en la región anorrectal $(20,23,24)$.

En cuanto a la edad, se determinó que la presencia de nódulos fue mayor a menor edad del paciente, similar a lo encontrado en el estudio cubano (6). Existen diferencias significativas respecto a la presencia de hiperplasia nodular de acuerdo con la indicación para la realización de la colonoscopia, lo que podría estar en relación con una mayor frecuencia de hiperplasia nodular en ciertas patologías específicas, incluida la alergia alimentaria; sin embargo, la asociación entre hiperplasia nodular y las causas específicas de la misma sobrepasa los objetivos de la presente investigación y debe investigarse a futuro.

La mayoría de pacientes con hiperplasia nodular linfoide en histología no presentó un patrón nodular durante la colonoscopia, lo que señala los diferentes patrones endoscópicos de lesión mucosa de la hiperplasia nodular linfoide (21-23), los cuales deben investigarse en futuros estudios. El VPP del hallazgo de nódulos fue bajo; al analizar el coeficiente de probabilidad positivo se observó que en pacientes con hiperplasia nodular existe solamente 2 veces más probabilidad de tener nódulos visibles durante la colonoscopia, por lo que el hallazgo nodular tiene pobre valor diagnóstico.

La especificidad alcanzada de la ausencia de nódulos en la colonoscopia fue alta, lo que se traduce en que la mayoría de pacientes sin hiperplasia nodular no presenta un patrón nodular, pero el VPN y el coeficiente de probabilidad negativo fueron bajos.

No se evaluó la utilidad de los hallazgos endoscópicos encontrados mediante nuevas técnicas endoscópicas tales como Narrow Band Imaging (NBI ${ }^{\oplus}$ de Olympus Medical Systems Corporation), Fuji Intelligent Color Enhancement (FICE ${ }^{\circledast}$ de Fujinon Corporation) o iSCAN (iSCAN ${ }^{\circledR}$ de Pentax) para el diagnóstico de hiperplasia nodular linfoide, por lo que hacen falta estudios para determinar su validez diagnóstica.

En cuando a la pobre validez global del hallazgo de nódulos durante la colonoscopia para el diagnóstico de hiperpla- 
sia nodular linfoide, consideramos oportuno abandonar la definición actual de hiperplasia nodular linfoide como un hallazgo de $>10$ nódulos visibles en endoscopía (1) por una definición histopatológica solamente, siendo la toma de biopsias indispensable durante la colonoscopia.

\section{Aspectos éticos}

El presente trabajo cumplió la normativa ética vigente tanto de nuestra institución como la internacional; no se realizó experimentación humana ni animal. Dado que se trata de un estudio descriptivo, no fue necesaria la obtención de consentimiento informado. Se ha mantenido estricta confidencialidad sobre la identidad de los participantes.

\section{Autoría}

Pablo Xavier Sempértegui-Cárdenas y José Francisco Cadena-León declaran autoría en el diseño y concepto del estudio, registro de datos, análisis estadístico, interpretación de datos y redacción del manuscrito.

Los coautores Karen Rubí Ignorosa-Arellano, Flora Elva Zárate-Mondragón, Roberto Cervantes-Bustamante, Jaime Alfonso Ramírez-Mayans, Celso Tomás CorcueraDelgado y Laura Becerril-Cholula declaran autoría en la revisión crítica del manuscrito por contenido intelectual importante.

\section{Fuentes de financiación}

No se recibió patrocinio de ningún tipo para llevar a cabo este artículo.

\section{Conflicto de intereses}

Los autores declaran no tener ningún conflicto de intereses.

\section{REFERENCIAS}

1. Mansueto P, Iacono G, Seidita A, D’Alcamo A, Sprini D, Carroccio A. Review article: intestinal lymphoid nodular hyperplasia in children--the relationship to food hypersensitivity. Aliment Pharmacol Ther. 2012;35(9):1000-9. https://doi.org/10.1111/j.1365-2036.2012.05062.x

2. Colarian J, Calzada R, Jaszewski R. Nodular lymphoid hyperplasia of the colon in adults: is it common? Gastrointest Endosc. 1990;36(4):421-2. https://doi. org/10.1016/s0016-5107(90)71092-9

3. Bharadhwaj G, Triadafilopoulos G. Endoscopic appearances of colonic lymphoid nodules: new faces of an old histopathological entity. Am J Gastroenterol. 1995;90(6):946-50.

4. Colón AR, DiPalma JS, Leftridge CA. Intestinal lymphonodular hyperplasia of childhood: patterns of presentation. J Clin Gastroenterol. 1991;13(2):163-6. https://doi. org/10.1097/00004836-199104000-00009

5. Gurkan OE, Yilmaz G, Aksu AU, Demirtas Z, Akyol G, Dalgic B. Colonic lymphoid nodular hyperplasia in childhood: causes of familial Mediterranean fever need extra attention. J Pediatr Gastroenterol Nutr. 2013;57(6):81721. https://doi.org/10.1097/MPG.0b013e3182a9083b

6. Silverio C, García W, Andrade M. Diagnósticos colonoscópicos más frecuentes en pediatría. Rev Cubana Pediatr. $2001 ; 73(1): 28-33$.

7. Khuroo MS, Khuroo NS, Khuroo MS. Diffuse duodenal nodular lymphoid hyperplasia: a large cohort of patients etiologically related to Helicobacter pylori infection. BMC Gastroenterol. 2011;11:36. https://doi. org/10.1186/1471-230X-11-36
8. Rubio-Tapia A, Hernández-Calleros J, Trinidad-Hernández S, Uscanga L. Clinical characteristics of a group of adults with nodular lymphoid hyperplasia: a single center experience. World J Gastroenterol. 2006;12(12):1945-8. https://doi.org/10.3748/wjg.v12.i12.1945

9. Canto J, Arista J, Hernández J. Hiperplasia linfoide nodular intestinal. Características clínico-patológicas en 11 casos [Nodular lymphoid hyperplasia of the intestine. Clinicopathologic characteristics in 11 cases]. Rev Invest Clin. 1990;42(3):198-203.

10. Olmez S, Aslan M, Yavuz A, Bulut G, Dulger AC. Diffuse nodular lymphoid hyperplasia of the small bowel associated with common variable immunodeficiency and giardiasis: a rare case report. Wien Klin Wochenschr. 2014 May;126(910):294-7. https://doi.org/10.1007/s00508-014-0525-5

11. Onbaşi K, Günşar F, Sin AZ, Ardeniz O, Kokuludağ A, Sebik F. Common variable immunodeficiency (CVID) presenting with malabsorption due to giardiasis. Turk J Gastroenterol. 2005;16(2):111-3.

12. Ajdukiewicz AB, Youngs GR, Bouchier IA. Nodular lymphoid hyperplasia with hypogammaglobulinaemia. Gut. 1972;13(8):589-95. https://doi.org/10.1136/gut.13.8.589

13. Hermans P, Huizenga K, Hoffman H, Brown A, Markowitz H. Dysgammaglobulinemia associated with nodular lymphoid hyperplasia of the small intestine. Am J Med. 1966;40(1):78-89. https://doi.org/10.1016/00029343(66)90189-6

14. Nazi N, Ladomenou F. Gastrointestinal manifestations of primary immune deficiencies in children. Int Rev Immunol. 
2018;37(2):111-118. https://doi.org/10.1080/08830185 .2017 .1365147

15. Postgate A, Despott E, Talbot I, Phillips R, Aylwin A, Fraser C. An unusual cause of diarrhea: diffuse intestinal nodular lymphoid hyperplasia in association with selective immunoglobulin A deficiency (with video). Gastrointest Endosc. 2009;70(1):168-9; discussion 169. https://doi. org/10.1016/j.gie.2009.03.004

16. Webster AD, Kenwright S, Ballard J, Shiner M, Slavin G, Levi AJ, Loewi G, Asherson GL. Nodular lymphoid hyperplasia of the bowel in primary hypogammaglobulinaemia: study of in vivo and in vitro lymphocyte function. Gut. 1977;18(5):364-72. https://doi.org/10.1136/gut.18.5.364

17. Levendoglu H, Rosen Y. Nodular lymphoid hyperplasia of gut in HIV infection. Am J Gastroenterol. 1992;87(9):1200-2.

18. Iacono G, Ravelli A, Di Prima L, Scalici C, Bolognini S, Chiappa S, Pirrone G, Licastri G, Carroccio A. Colonic lymphoid nodular hyperplasia in children: relationship to food hypersensitivity. Clin Gastroenterol Hepatol. 2007;5(3):361-6. https://doi.org/10.1016/j. cgh.2006.12.010

19. Schwartz DC, Cole CE, Sun Y, Jacoby RF. Diffuse nodular lymphoid hyperplasia of the colon: polyposis syndrome or normal variant? Gastrointest Endosc. 2003;58(4):630-2.

20. Kuper CF. Histopathology of mucosa-associated lymphoid tissue. Toxicol Pathol. 2006;34(5):609-15. https://doi. org/10.1080/01926230600867735

21. Smith MB, Blackstone MO. Colonic lymphoid nodules: another cause of the red ring sign. Gastrointest Endosc. 1991;37(2):206-8. https://doi.org/10.1016/s00165107(91)70692-5

22. Straub RF, Wilcox CM, Schwartz DA. Variable endoscopic appearance of colonic lymphoid tissue. J Clin Gastroenterol. 1994;19(2):158-64; discussion 164-5. https://doi.org/10.1097/00004836-199409000-00018

23. Molaei M, Kaboli A, Fathi AM, Mashayekhi R, Pejhan $S$, Zali MR. Nodular lymphoid hyperplasia in common variable immunodeficiency syndrome mimicking familial adenomatous polyposis on endoscopy. Indian J Pathol Microbiol. 2009;52(4):530-3. https://doi. org/10.4103/0377-4929.56152

24. Bayraktar Y, Ersoy O, Sokmensuer C. The findings of capsule endoscopy in patients with common variable immunodeficiency syndrome. Hepatogastroenterology. 2007;54(76):1034-7.
25. Rambaud JC, De Saint-Louvent P, Marti R, Galian A, Mason DY, Wassef M, Licht H, Valleur P, Bernier JJ. Diffuse follicular lymphoid hyperplasia of the small intestine without primary immunoglobulin deficiency. Am J Med. 1982;73(1):125-32. https://doi. org/10.1016/0002-9343(82)90938-x

26. Tomita S, Kojima M, Imura J, Ueda Y, Koitabashi A, Suzuki Y, Nakamura Y, Mitani K, Terano A, Fujimori T. Diffuse nodular lymphoid hyperplasia of the large bowel without hypogammaglobulinemia or malabsorption syndrome: a case report and literature review. Int J Surg Pathol. 2002;10(4):297-302. https:// doi. org/10.1177/106689690201000411

27. Ranchod M, Lewin KJ, Dorfman RF. Lymphoid hyperplasia of the gastrointestinal tract. A study of 26 cases and review of the literature. Am J Surg Pathol. 1978;2(4):383-400. https:// doi.org/10.1097/00000478-197812000-00005

28. Chiaramonte C, Glick SN. Nodular lymphoid hyperplasia of the small bowel complicated by jejunal lymphoma in a patient with common variable immune deficiency syndrome. AJR Am J Roentgenol. 1994;163(5):1118-9. https://doi.org/10.2214/ajr.163.5.7976886

29. Schaefer PS, Friedman AC. Nodular lymphoid hyperplasia of the small intestine with Burkitt's lymphoma and dysgammaglobulinemia. Gastrointest Radiol. 1981;6(4):325-8. https://doi.org/10.1007/BF01890278

30. Monsanto P, Lérias C, Almeida N, Lopes S, Cabral JE, Figueiredo P, Silva M, Julião M, Gouveia H, Sofia C. Intestinal nodular lymphoid hyperplasia and extraintestinal lymphoma--a rare association. Acta Gastroenterol Belg. 2012;75(2):260-2.

31. Jonsson OT, Birgisson S, Reykdal S. Resolution of nodular lymphoid hyperplasia of the gastrointestinal tract following chemotherapy for extraintestinal lymphoma. Dig Dis Sci. 2002;47(11):2463-5. https://doi. org/10.1023/a:1020547723325

32. Rubio CA. Nonprotruding colorectal neoplasms: epidemiologic viewpoint. World J Surg. 2000;24(9):1098-103. https://doi.org/10.1007/s002680010147

33. Zahmatkeshan M, Fallahzadeh E, Najib K, Geramizadeh B, Haghighat M, Imanieh MH. Etiology of lower gastrointestinal bleeding in children: a single center experience from southern iran. Middle East J Dig Dis. 2012;4(4):216-23. 